\title{
Textile Rectenna for RF Energy Harvesting
}

\author{
Fábio Silva \\ Instituto Superior de Engenharia de Lisboa \\ Instituto de Telecomunicações \\ Aveiro, Portugal \\ fabiossilva21@gmail.com
}

\author{
Rita Almeida \\ Instituto Superior de Engenharia de Lisboa \\ Instituto de Telecomunicações \\ Aveiro, Portugal \\ ritafrancaalmeida@gmail.com
}

\author{
Carolina Gouveia \\ Universidade de Aveiro \\ Instituto de Telecomunicações \\ Aveiro, Portugal \\ carolina.gouveia@ua.pt
}

\author{
Caroline Loss \\ FibEnTech Research Unit \\ University of Beira Interior \\ Covilhã, Portugal \\ carol@ubi.pt
}

\author{
Pedro Pinho \\ Instituto Superior de Engenharia de Lisboa \\ Instituto de Telecomunicações \\ Aveiro, Portugal \\ pedro.pinho@isel.pt
}

\author{
Daniel Belo \\ Universidade de Aveiro \\ Instituto de Telecomunicações \\ Aveiro, Portugal \\ a35842@ua.pt
}

\begin{abstract}
Wearable devices are part of many people's lives, however the batteries they need are a major limitation. In order to solve this problem, an energy harvesting system was studied and implemented, to replace the battery usage and thus enhance the device portability. It is composed by two antennas, one for transmitting and the other for receiving, and a rectifier circuit. The system was completely developed in textile, allowing the integration into the user's clothing.
\end{abstract} tion.

Index Terms-Wearable, WPT, RF-dc, Antennas, Rectifica-

\section{INTRODUCTION}

Currently, the power limitation in wearable devices is a very critical factor, since it is require to use batteries to power these devices which implies a battery changes. This is inconvenient, especially in devices integrated in clothing, as it is necessary to open the device to perform the replacement. Considering this limitation, there is an increasing demand and interest in using more sustainable and ecological techniques, that are capable of improving various aspects of these devices, such as longevity, weight, etc... One of these techniques is Wireless Power Transmission (WPT). In this work, a WPT system is proposed. All the systems components, being the transmitting (Tx) and receiving $(\mathrm{Rx})$ antennas and a rectifier circuit are implemented using textile materials. The system operates in the far field region, at $5.8 \mathrm{GHz}$ and using the microwave method, [1].

In the literature, there are many papers about textile antennas and RF-dc circuits. For example, in [2] the authors present an RF-dc circuit made in rigid substrate and only present simulated results. In [3] the authors present an antenna for wearable devices operating $5.8 \mathrm{GHz}$. However this antenna was made using FR-4 as substrate and the resonance frequency deviated, resonating at $5.96 \mathrm{GHz}$. In [4] a system for energy harvesting is proposed, using a textile antenna and a rectifier circuit built in a flexible substrate, not textile. In our work, we proposed an energy harvesting system fully implemented in textile substrate, which is the main difference between this work and the others presented. Thus, this paper is a contribution for the development and construction of a textile system for WPT, with passive components welded directly to the textile material and circuit vias fully implemented in textile using conductive threads. The proposed system contains two antennas and one RF-dc circuit all made in textile.

The system works as the following: a signal is transmitted with a carrier frequency of $5.8 \mathrm{GHz}$. This signal will be received by the receiver that, with the aid of an RF-dc circuit, will convert the RF signal into an available DC power, which will supply a given load. Textile materials were selected to build the system components and thus allow the fully integration into the user's clothing, in a discreet and comfortable way. This way, the wearable device is powered through WPT, as illustrated in Figure 1.
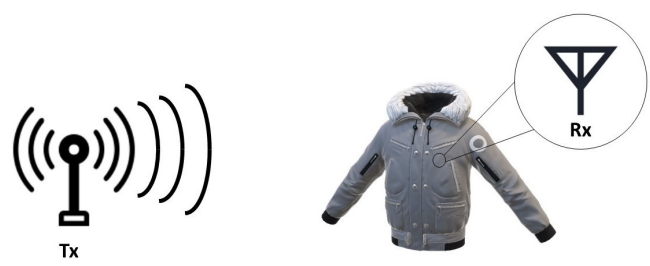

Fig. 1. Representation of the WPT concept.

This paper is divided as follows: first, the antenna design and results are presented in Section II. Then, the RF-dc circuit is presented in Section III, where the design is presented, as well as the simulated and measured results obtained. Finally, Section IV presents the main conclusions.

\section{Antenna}

The textile antenna was simulated using the software CST Studio Suite. For the substrate, a dielectric weft-knitted fabric called PDE Black (Borgstena Textile Portugal, Portugal), with a relative permittivity constant of 1.385 , loss tangent of 0.008 , and a thickness of $0.75 \mathrm{~mm}$, was used. For the conductive parts, an e-textile named Pure Copper Taffeta Fabric (Less 
EMF, USA) [5], with conductivity of $62500 \mathrm{~S} / \mathrm{m}$ and a thickness of $0.08 \mathrm{~mm}$, was used.

The geometry of the textile antenna designed is shown in Fig. 2, and its dimensions are presented in Table I. This element is composed of a rectangular radiating patch and a $50 \Omega$ transmission line that feeds the radiating patch in a $50 \Omega$ point using the inset feed method.

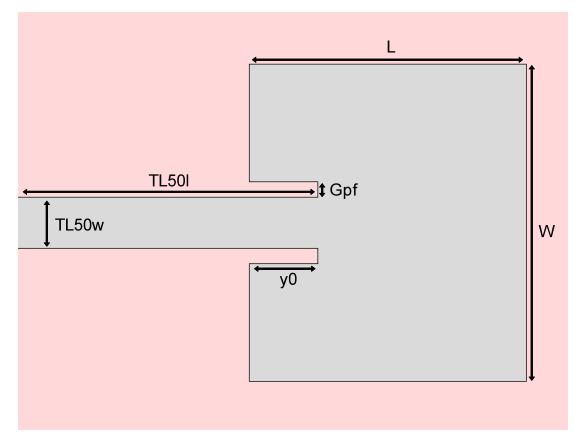

Fig. 2. Geometry of the designed textile antenna.

TABLE I

TEXTILE ANTENNA DIMENSIONS.

\begin{tabular}{|c|c|}
\hline Variable & Value [mm] \\
\hline L, W & $20.81,23.43$ \\
\hline TL501, TL50w & $3.77,22.49$ \\
\hline Gpf, y0 & $1.1,4.17$ \\
\hline
\end{tabular}

After their simulation, the antenna was built using the laminating manufacturing technique. It consists of assembling the components with the thermal adhesive sheet through a simple ironing operation. In this case, a continuous web called Fixorete Contínuo (Jaú Têxteis, Portugal) was used. To ensure the correctly geometrical dimensions of the antenna, all parts were cut by laser cutting machine. As the WPT system requires one transmitter and one receiver antennas, two prototypes were built.

The $S_{11}$ parameters are presented in Fig. 3, where the antenna 1 has a value of $-20 \mathrm{~dB}$ and the antenna 2 has a value of $-23.3 \mathrm{~dB}$ at $5.8 \mathrm{GHz}$.

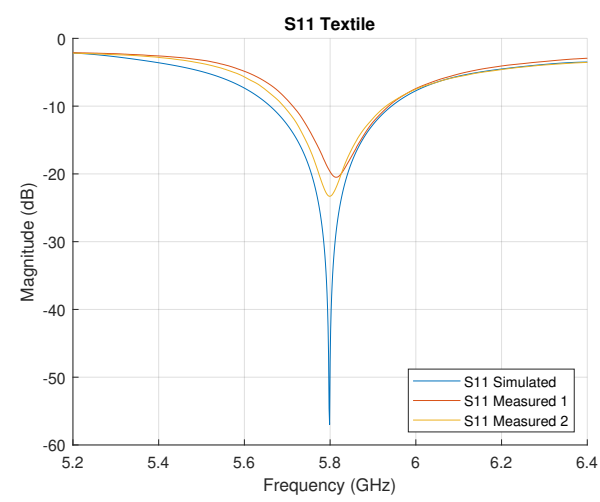

Fig. 3. Simulated and measured $S_{11}$ parameter of both antennas.
By observing Fig. 3, it is also possible to observe a slight difference in the $S_{11}$ parameter of both antennas. These small differences between the results are expected due to imprecisions related with the manual manufacturing process, and the flexibility of the textile materials.

An example of the built antenna can be seen in Fig. 4.

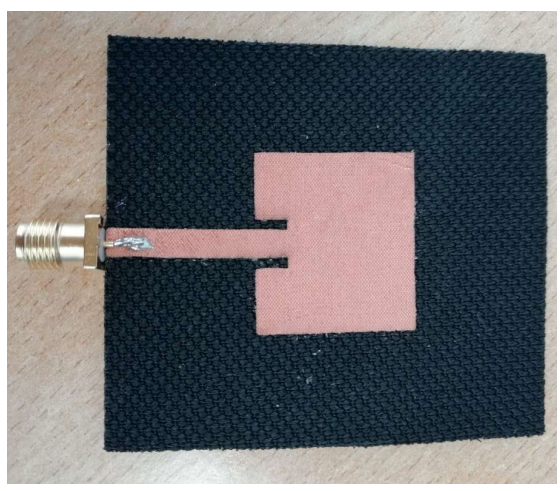

Fig. 4. Textile antenna.

Once the $S_{11}$ was measured and the resonance frequency was confirmed at $5.8 \mathrm{GHz}$, the radiation pattern of the antenna 1 was measured in an anechoic chamber, and the results can be seen in Fig. 5 .

It should be noted that the flexibility of the fabric causes changes in its electromagnetic properties, a phenomenon that was observed during the measurements of the $S_{11}$ parameters of the antennas, having easily deviated its resonance frequency during the measurement process. In order to avoid these changes, and as indicated in [6], the antenna should be placed in a part of the body unlikely to bend. For that, we propose the torso, that way it is possible to reduce any effects the antenna may suffer by bending it.

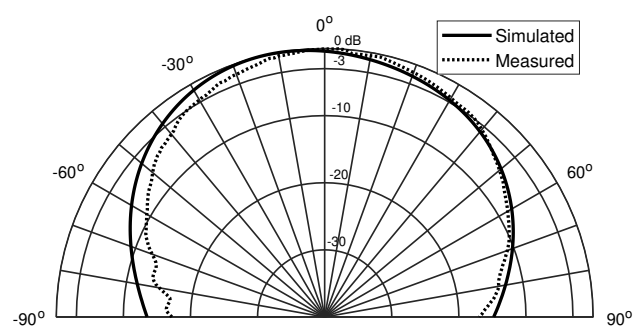

Fig. 5. Vertical Radiation Pattern.

As it can be seen in the radiation pattern, the measured results are very close to the simulated ones. The simulated antenna has a half-power beam width of $76^{\circ}$ and the manufactured antenna of $72^{\circ}$, resulting in a slightly more directive antenna.

\section{RF-DC CIRCUIT}

The RF-dc circuit was simulated in the Advanced Design System (ADS) Software by Keysight. Also, aiming for the full integration of the WTP system into clothes, the RF-dc circuit was simulated using the same textile materials used in 
the construction of the textile antenna, presented in Section II.

For the RF-dc Circuit a Karthaus-Fischer cascade voltage multiplier [7], was used with certain modifications, which can be seen in Fig. 6. For the diodes, the Schottky SMS7630079LF [8] was used and for the load it was used a red LED [9]. However, during the simulations, the load was modeled as a $2.125 \mathrm{k} \Omega$ resistor. This value resulted from the LED pulling approximately $800 \mu \mathrm{A}$ at a voltage of $1.7 \mathrm{~V}$, as indicated in the datasheet [9].

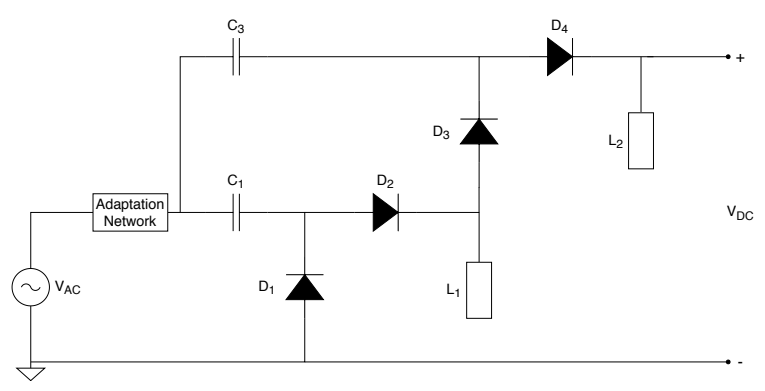

Fig. 6. Modified Karthaus-Fischer voltage multiplier.

First it was added a matching network in order to obtain an acceptable $S_{11}$ parameter at the desired frequency and to provide maximum power transfer between the antenna and the RF-dc circuit. This matching network follows a $\mathrm{T}$ configuration, made with two standard transmission lines and one short-circuited stub. The last modification was the introduction of two open stubs $\left(\mathrm{L}_{1}\right.$ and $\left.\mathrm{L}_{2}\right)$ in place of the output capacitors, in order to spare components. These stubs have a length of $\lambda / 4$ at $5.8 \mathrm{GHz}$, in order to short circuit the fundamental and the even-order harmonics.

The simulation was done with the Harmonic Balance controller, in order to simulate the effect of the harmonics created by the diodes have on the circuit. The circuit was simulated from the fundamental frequency $(5.8 \mathrm{GHz})$ to the 5th harmonic $(29 \mathrm{GHz})$.

After using the schematic model of the circuit to achieve the desired voltage, the layout tool was used to create the electromagnetic model of the circuit and simulate it using the Momentum simulator provided with ADS. This approach provides more realistic results which enable a reliable optimization. These results can be seen in Fig. 7, where the desired voltage was pointed out.

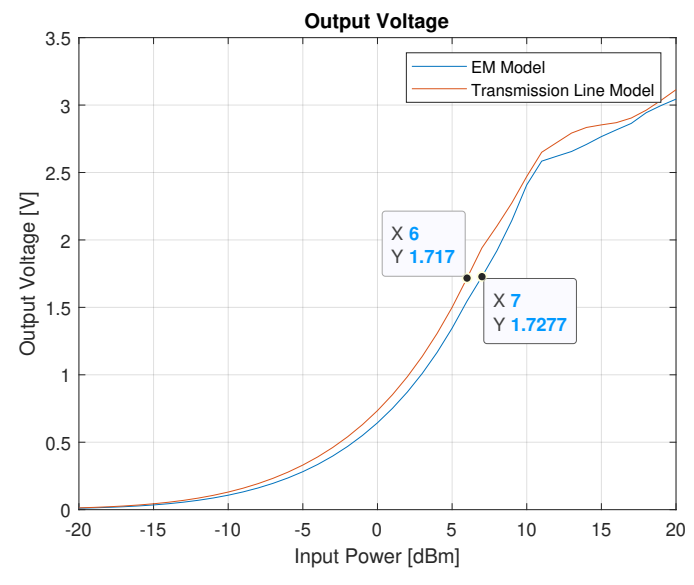

Fig. 7. Simulated RF-dc circuit output voltage.

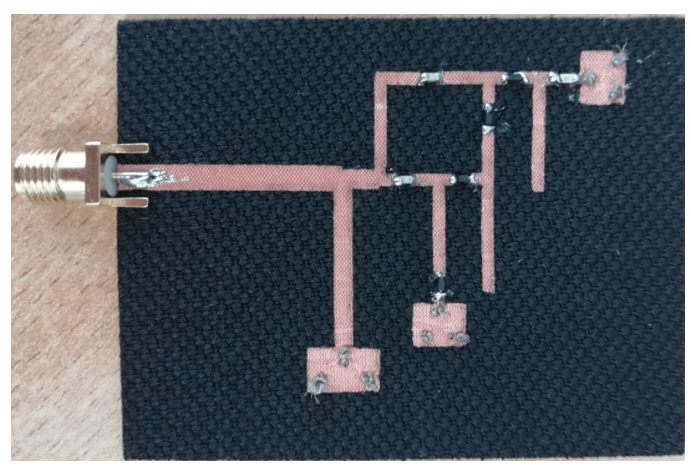

Fig. 8. Final RF-dc textile circuit.

After its simulation, the circuit was constructed by using the laminating manufacturing technique, which can be seen in Fig. 8. To maintain the flexibility of the circuit, the vias were made using commercially available conductive threads, made of polyester silver coated, called Silverpam 250 (Tibtech, France) [10]. The vias were constructed through a seams operation, connecting the stubs to their ground plane. Finally, the passive components and the SMA connector were welded to the conductive fabric. This way, the whole system, except the passive components, was fully implemented on textile.

The $S_{11}$ of the manufactured textile circuit was measured with an input power of $7 \mathrm{dBm}$, which can be seen in Fig. 9 along with the simulated results.

Finally, the output voltage was measured, which can be seen in Fig. 10. Depending on the input power an LED has, its resistance changes, this can be confirmed by looking at the forward current versus forward voltage graph provided in the datasheet [9], and noting it is not a linear relationship. Since the measured circuit used an LED as a load, and the simulations used a $2.125 \mathrm{k} \Omega$ resistor as a load for all input powers, the measured output voltages should not follow the simulated output voltages. 


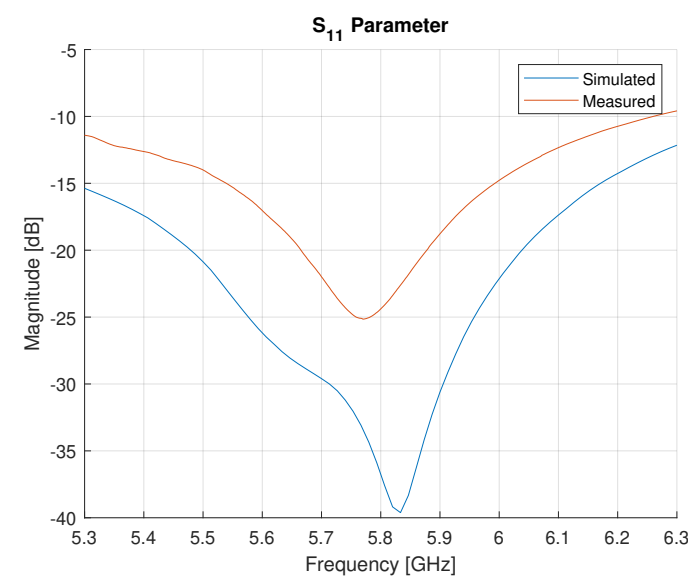

Fig. 9. Simulated and measured $S_{11}$ parameter of the circuit.

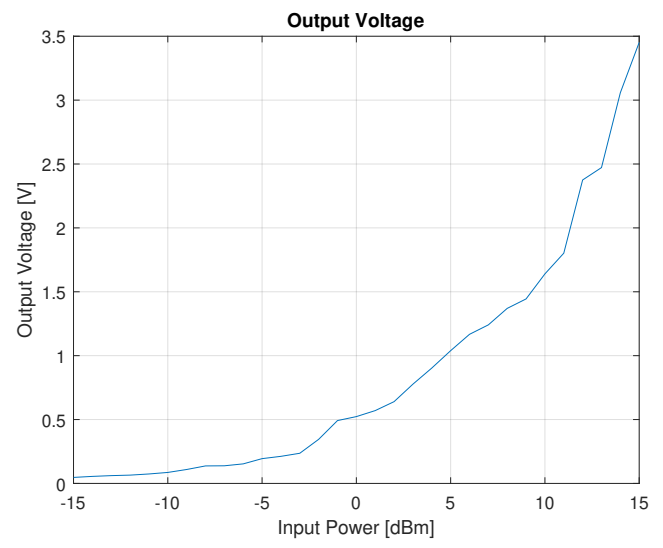

Fig. 10. Measured Output Voltage.

In Figure 11, it is possible to observe the entire WPT system, where the transmitting antenna is represented by the blue rectangle, and the receiving antenna and the RF-dc circuit are represented by the yellow rectangle.

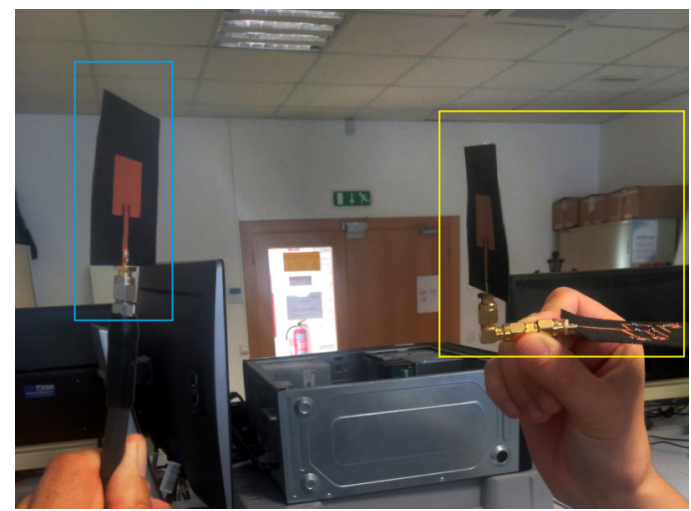

Fig. 11. Textile WPT system.

\section{CONCLUSION}

In this work, both antennas and the RF-dc circuit were successfully implemented using textile materials. The man- ufacturing of a textile circuit is challenging given that the textile presents some difficulties implementing circuits since it is fragile and flexible. The major issue is to create good and stable connections between the textile parts and the rigid interfaces, as the SMA connector and the passive elements. For instance, the welding process using high temperatures can burn the fabric, damaging it. Despite the challenge, the antenna and circuit have been successfully implemented on textiles, achieving good results, closed to the simulated ones.

This is ongoing research and the future work may include: implementing the receiver antenna and the rectifier circuit in the same substrate would reduce some losses in the system, creating an System on Substrate (SoS). Implementing the same concept with lower frequencies would improve the range of the project since lower frequencies have lower losses in free space. The transmitter antenna could be implemented in a rigid substrate with other geometry, which could improve the transmission. Finally, studying and using other types of rectifiers could prove beneficial to this work.

\section{ACKNOWLEDGMENT}

This work is funded by FCT/MCTES through national funds and when applicable co-funded EU funds under the project UIDB/50008/2020-UIDP/50008/2020 and UIDB/00195/2020. This work is also funded by the Fundação para a Ciência e Tecnologia (FCT) through Fundo Social Europeu (FSE) and by Programa Operacional Regional do Centro under the PhD grant SFRH/BD/139847/2018.

\section{REFERENCES}

[1] R. S. Lakshmi, "RF Energy Harvesting for Wireless Devices," International Journal of Engineering Research and Development, pp. 39-52, 2015.

[2] A. Taybi, A. Tajmouati, J. Zbitou, A. Errkik, and M. Latrach, "Design of a new 5.8 GHz RF-DC rectifier structure for wireless power transmission," in 2017 International Conference on Wireless Technologies, Embedded and Intelligent Systems (WITS), 2017, pp. 1-4.

[3] S. Manna, T. Bose, and R. Bera, "Patch Antenna of Self Similar Structure for Wearable Devices Operating Around $5.8 \mathrm{GHz}$," in 2019 International Conference on Opto-Electronics and Applied Optics (Optronix), 2019, pp. 1-3.

[4] A. Costanzo, D. Masotti, and M. Del Prete, "Wireless power supplying flexible and wearable systems," in 2013 7th European Conference on Antennas and Propagation (EuCAP), 2013, pp. 2843-2846.

[5] "Electromagnetic Field Shielding Fabrics - High Tech and Industrial," https://www.lessemf.com/fabric4.html\#1212, accessed: September 2020.

[6] F. Gemperle, C. Kasabach, J. Stivoric, M. Bauer, and R. Martin, "Design for wearability," Digest of Papers. Second International Symposium on Wearable Computers, pp. 116-122, 1998.

[7] X. S. Loo, K. S. Yeo, J. Yang, C. H. Lee, R. Zhao, and M. Z. Win, "High voltage energy harvesters," in Advanced Electronic Circuits Principles, Architectures and Applications on Emerging Technologies, IntechOpen 2018.

[8] "SMS7630-079LF SMD Diode Datasheet," https://pt.mouser.com/datasheet/2/472/Surface_Mount_Schottky_Diodes_ 200041AD-708941.pdf, accessed: July 2020.

[9] "APHD1608LSURCK SMD LED Datasheet," https://pt.mouser.com/datasheet/2/216/APHD1608LSURCK1100899.pdf, accessed: July 2020.

[10] "SILVERPAM 250 : Grafted antibacterial silver thread," https://www.tibtech.com/smartshop/index.php?id_product=80\&controller= product, accessed: September 2020. 